\title{
Dosimetric accuracy of a cross-calibration coefficient for plane-parallel ionization chamber obtained in low-energy electron beams using various cylindrical dosimeters
}

\author{
Kinga POLACZEK-GRELIKa, ${ }^{a}$, Aneta KAWA-IWANICKA ${ }^{\mathrm{a}}$, Łukasz MICHALECKI ${ }^{\mathrm{a}}$ \\ ${ }^{a} N U$-Med Cancer Diagnosis and Treatment Centre Katowice, Poland \\ ${ }^{*}$ E-mail address: kinga.polaczek-grelik@nu-med.pl
}

\begin{abstract}
Introduction: The accuracy of the cross-calibration procedure depends on ionization chamber type, both used as reference one and under consideration. Also, the beam energy and phantom medium could influence the precision of cross calibration coefficient, resulting in a systematic error in dose estimation and thus could influence the linac beam output checking. This will result in a systematic mismatch between dose calculated in treatment planning system and delivered to the patient.

Material and methods: The usage of FC65-G, CC13 and CC01 thimble reference chambers as well as 6, 9, and $15 \mathrm{MeV}$ electron beams has been analyzed. A plane-parallel PPC05 chamber was calibrated since scarce literature data are available for this dosimeter type. The influence of measurement medium and an effective point of measurement (EPOM) on obtained results are also presented.

Results: Dose reconstruction precision of $\sim 0.1 \%$ for PPC05 chamber could be obtained when cross-calibration is based on a thimble CC13 chamber. $\mathrm{N}_{\mathrm{d}, \mathrm{w}, \mathrm{Q} \text {,ross }}$ obtained in beam $\geq 9 \mathrm{MeV}$ gives $0.1-0.5 \%$ precision of dose reconstruction. Without beam quality correction, $15 \mathrm{MeV} \mathrm{N}$ d,w,Qcross is $10 \%$ lower than Co-60 $\mathrm{N}_{\mathrm{d}, \mathrm{w}, 0}$. Various EPOM shifts resulted in up to $0.6 \%$ discrepancies in $\mathrm{N}_{\mathrm{d}, \mathrm{w}, \mathrm{Q} \text { cross }}$ values.

Conclusions: Ionization chamber with small active volume and tissue-equivalent materials supplies more accurate crosscalibration coefficients in the range of $6-15 \mathrm{MeV}$ electron beams. In the case of 6 and $9 \mathrm{MeV}$ beams, the exact position of an effective point of measurement is of minor importance. In-water cross-calibration coefficient can be used in a solid medium without loss of dose accuracy.
\end{abstract}

Key words: cross calibration; low-energy electron beams; effective point of measurement; PPC05 ionization chamber; dosimetry in radiotherapy.

\section{Introduction}

Plane-parallel ionization chambers in clinical practice are used both for relative (e.g. percentage depth dose - PDD) and absolute (e.g. absorbed dose, beam output) measurements. These dosimeters are particularly useful in high dose gradient regions and for minimizing the uncertainty of effective chamber point positioning ${ }^{1}$ due to the specific construction, i.e. "pancake" shape of air cavity and a small distance between the electrodes. Moreover, this kind of detector is recommended for electron beam quality control in all leading dosimetric reports, i.e. IAEA TRS-398, AAPM TG-51, DIN 6800-2 and IPEM codes of practice $(\mathrm{CoP}){ }^{1-4}$ Their use is advised for absolute measurements of low-energy electron beams $(\mathrm{E} \leq 10 \mathrm{MeV}){ }^{1-3}$ whereas for high-energy electron beam dosimetry it is the user's choice, often made for practical reasons. On the other hand, the use of a cylindrical chamber is recommended for at least $\mathrm{E}_{0}=9.5 \mathrm{MeV}$ electron energy. ${ }^{3}$ Nevertheless, some publications, e.g. ${ }^{\mathbf{5}, \mathbf{6}}$, suggest that thimble chambers are suitable for use in all electron beams clinically available.

It is of common practice to calibrate plane-parallel ionization chambers, similar to thimble chambers, in reference $\left({ }^{60} \mathrm{Co}\right)$ photon beam by Secondary Standard Dosimetry Laboratory (SSDL) and derive calibration coefficients $\mathrm{N}_{\mathrm{d}, \mathrm{w}, \mathrm{Q} \text {. }}$. However, to maintain the dose estimation uncertainty at the lowest possible level (mainly due to beam quality correction factor ${ }^{2}$ ), the electron beam of reference quality should be used instead. As presented in ${ }^{8}$, using $22 \mathrm{MeV}$ electron beam for $\mathrm{N}_{\mathrm{d}, \mathrm{w}}$ calibration purposes, the differences could be minimalized below $0.5 \%$ (average of $0.1 \%$ ) precision recommended by the IAEA $^{1}$ in comparison with $\mathrm{N}_{\mathrm{d}, \mathrm{w}, \mathrm{Q} 0}$. Moreover, some studies are concentrated on the determination of chamber type-specific wall perturbation correction factors to enable the electron beam dosimetry without the necessity of performing a cross calibration procedure at all. ${ }^{3}$ 
Formalisms regarding the procedure of ionization chamber cross calibration provide the data for arbitrarily chosen electron beam of the quality characterized by $\mathrm{R}_{50}=7.5 \mathrm{~g} / \mathrm{cm}^{2}$, which means the average energy at the phantom surface of $\mathrm{E}_{0}=18 \mathrm{MeV} .^{\mathbf{1 , 2}}$ This quality is described as $\mathrm{Q}_{\text {int. }}{ }^{1}$ However, some commercially available linear accelerators do not generate electron beams with energies of $18 \mathrm{MeV}$ and above. In such a situation the user is forced to perform the cross-calibration procedure for electron beams used in clinical practice, even when the energy is below the recommended one $(\leq 16 \mathrm{MeV})$.

There are several issues that have to be decided to perform the cross-calibration procedure with high precision, i.e., the type of cylindrical reference chamber most appropriate for measurement conditions, the influence of electron beam energy on the precision and uncertainty of cross-calibration coefficients, the phantom medium, i.e., water vs. solid material radiologically water equivalent. Absolute dose measurement in a water reference medium is recommended. ${ }^{\mathbf{1 - 3}}$ However, the weekly check of beam output (cGy/MU) constancy is often performed in a solid phantom. Therefore, it should be decided whether cross-calibration coefficient should be determined in water or could be done in a solid medium routinely used.

There are also different approaches to the issue of the effective point of measurement (EPOM). Many publications, e.g. ${ }^{10,11}$ and references therein, were devoted to the determination of EPOM either experimentally or analytically for various types of ionization chambers in electron and photon beams. However, for practical reasons, the dosimetric reports allow for using a simplified approach. For cylindrical chambers (regardless of their type) used for electron beam measurements, EPOM should be shifted by $0.5 \mathrm{r}_{\text {cyl }}$ according to TRS-398, DIN 6800-2 and TG70 CoPs ${ }^{\mathbf{1 , 4 5}}$ or by $0.6 \mathrm{r}_{\text {cyl }}$ according to TG-51 and IPEM CoPs ${ }^{\mathbf{2 , 3}}$. In the case of plane-parallel dosimeters, it is placed on the inner surface of the entrance window. However, various studies have shown that the EPOM position depends on the detector construction, regardless of the beam energy, field size, and measuring depth. ${ }^{\mathbf{1 0 - 1 2}}$ Monte Carlo simulations have shown that, depending on the method of EPOM determination, $0.5 \mathrm{r}_{\mathrm{cyl}}$ shift is correct or is too large, resulting in a systematic error ${ }^{\mathbf{1 3 , 1 4}}$ of ionization chamber readings. The smaller is the size of a thimble chamber, the smaller should be $\Delta z$ the shift of EPOM ${ }^{\mathbf{1 0 , 1 4}}$. However, for plane-parallel chambers, the lower was the electron beam energy, the larger EPOM shift was observed, as reported in ${ }^{\mathbf{1 3 , 1 5}}$. Moreover, such effect was more pronounced in the case of thin entrance window and narrow guard ring of the chamber. Therefore the values recommended in dosimetric protocols mentioned above seem to be the maximal values for ionization chambers used nowadays in clinical practice.

Therefore, the purpose of the presented study was to discuss all the above-mentioned issues and their quantitative influence on the precision of obtained $\mathrm{N}_{\mathrm{d}, \mathrm{w}}$ cross-calibration coefficients and absolute dose measurements results. Three types of cylindrical ionization chambers, which differ in an active volume, were used as reference dosimeters. Electron beams of three nominal energies (6, 9 and $15 \mathrm{MeV}$ ) were applied, and measurements were performed both in water and in solid phantom RW3. Measurements were done using EPOM shifts recommended in the TRS-398 report $^{1}$ as well as using those experimentally determined. Plane-parallel ionization chamber PPC05 has been cross-calibrated since this dosimeter is often used in clinical practice, but scarce data in the literature, including dosimetric protocols, could be found.

\section{Material and Methods}

The cross-calibration procedure of a plane-parallel ionization chamber was carried out using the Elekta Synergy linear accelerator equipped with a 160-leaf (MLC) Agility collimator. Electron beams of three nominal energies: 6, 9, and $15 \mathrm{MeV}$ were used. These are characterized by the parameter of $\mathrm{R}_{50}=2.5$ $\mathrm{g} / \mathrm{cm}^{2}, 3.5 \mathrm{~g} / \mathrm{cm}^{2}$ and $6.0 \mathrm{~g} / \mathrm{cm}^{2}$, and corresponding $Z_{\text {ref }}$ (obtained in accordance with TRS-398 report ${ }^{1}$ ) depths of $1.4 \mathrm{~g} / \mathrm{cm}^{2}, 2.1$ $\mathrm{g} / \mathrm{cm}^{2}$ and $3.5 \mathrm{~g} / \mathrm{cm}^{2}$, respectively. The ionization chambers were sequentially placed in water Blue Phantom 2 (IBA Dosimetry $\mathrm{GmbH}$ ), and the ionization measurements were performed for 100 monitor units (MU) each time. The measuring setup consisted of a reference-class electrometer DOSE 1 (IBA Dosimetry $\mathrm{GmbH}$ ) and a currently used ionization chamber. The set of studied dosimeters is described below in detail. Afterward, the measurements were repeated in a solid phantom SP34 (IBA Dosimetry $\mathrm{GmbH}$ ), made of RW3 material (98\% polystyrene + $2 \% \mathrm{TiO}_{2}$ ), in order to investigate the influence of the phantom medium on cross-calibration coefficient $\mathrm{N}_{\mathrm{d} \text {,w, Qcross }}$ values.

\subsection{Ionization chambers}

The guidelines of TRS-381 report ${ }^{16}$ regarding the elements of an ideal plane-parallel ionization chamber, discussed also in ${ }^{\mathbf{1 7}}$, allow for minimizing the perturbation effects, which subsequently enables a more precise result of dose estimation based on the ionization chamber measurements. The diameterto-height ratio $(\mathrm{D} / \mathrm{H})$ of the air cavity should be large, preferably $\geq 5$ according to $^{3}$, the width of a guard ring should not be smaller than 1.5 of air cavity height and the height $(\mathrm{H})$ itself should not be larger than $2 \mathrm{~mm}$. Such construction is perceived as a "wellguarded"16 and recommended for absolute dosimetry of electron beams. Additionally, the collecting electrode diameter should not exceed $20 \mathrm{~mm}^{3}$ The data characterizing several commonly available plane-parallel chambers (shown in Table 1) indicate that some types of clinically used dosimeters do not meet these criteria, and a variety of constructions are made according to them. Moreover, the differences in so-called Markus-type chambers are often large (see: first 3 rows of Table 1), and correction factors given in dosimetric reports for Markus chamber are not suitable even for Markus-type chambers (e.g., PPC05) due to fundamental differences in construction. Therefore, the end-user should be aware of the potentially increased uncertainty of dose measurement (even $2 \%$ ) when using the ionization chamber, which does not meet the TRS-381 criteria. 
Table 1. The general data regarding the exemplary commercially available plane-parallel ionization chambers. Characteristics based on IBA Dosimetry GmbH and Best Medical products information.

\begin{tabular}{|c|c|c|c|c|c|}
\hline $\begin{array}{l}\text { Ionization } \\
\text { chamber }\end{array}$ & $\begin{array}{c}\text { Air cavity diameter }(\mathrm{D}) \\
{[\mathrm{mm}]}\end{array}$ & $\begin{array}{c}\text { Air cavity height }(\mathrm{H}) \\
{[\mathrm{mm}]}\end{array}$ & $\begin{array}{l}\mathrm{D} / \mathrm{H} \\
\text { ratio }\end{array}$ & $\begin{array}{c}\text { Guard ring width } \\
{[\mathrm{mm}]}\end{array}$ & Entrance window \\
\hline Markus & 5.3 & 2.0 & 2.65 & 0.1 & $0.03 \mathrm{~mm} \mathrm{PE} * * *, 2.76 \mathrm{mg} / \mathrm{cm}^{2}$ \\
\hline Advanced Markus & 5.0 & 1.0 & 5 & 2.0 & $0.03 \mathrm{~mm}$ PE, $2.76 \mathrm{mg} / \mathrm{cm}^{2}$ \\
\hline PPC05 & 10 & 0.5 & 20 & 3.5 & $1 \mathrm{~mm} \mathrm{C}-552^{*}, 176 \mathrm{mg} / \mathrm{cm}^{2}$ \\
\hline Roos & 15 & 2.0 & 7.5 & 4.0 & $1 \mathrm{~mm}$ acryl, $118 \mathrm{mg} / \mathrm{cm}^{2}$ \\
\hline PPC40 & 16 & 2.0 & 8 & 4.0 & $1 \mathrm{~mm} \mathrm{PMMA}^{* *}, 118 \mathrm{mg} / \mathrm{cm}^{2}$ \\
\hline NACP & 10 & 2.0 & 5 & 3.0 & $0.6 \mathrm{~mm}$ mylar folie and graphite, $104 \mathrm{mg} / \mathrm{cm}^{2}$ \\
\hline
\end{tabular}

Table 2. Characteristics of cylindrical ionization chambers used in the presented study, based on the manufacturer's (IBA Dosimetry GmbH) description.

\begin{tabular}{|c|c|c|c|c|c|}
\hline Ionization chamber & Air cavity diameter $[\mathrm{mm}]$ & Air cavity length [mm] & Active volume $\left[\mathrm{cm}^{3}\right]$ & Wall material & Central electrode material \\
\hline FC65-G & 6.2 & 23.1 & 0.65 & Graphite & Aluminum \\
\hline $\mathrm{CC} 13$ & 6.0 & 5.8 & 0.13 & C-552 & C-552 \\
\hline $\mathrm{CC} 01$ & 2.0 & 3.6 & 0.01 & C-552 & Steel \\
\hline
\end{tabular}

Vented ionization chambers were used in the presented study. Cylindrical (thimble) ionization chambers: Farmer type FC65-G and two compact chambers $\mathrm{CC} 13$ and $\mathrm{CC} 01$, were used as the reference dosimeters. Although $\mathrm{CC} 01$ dosimeter is not recommended as a reference chamber ${ }^{18}$ due to the high- $Z$ material of the central electrode and a very small active volume, it was involved in this study to compare the results obtained using chambers with different central electrode's materials (see: Table 2) and different sizes of the active volume. The differences in air cavity dimensions and wall/electrodes materials between cylindrical chambers (see: Table 2) make difficult to decide arbitrarily (only on the base of the manufacturer specification), which of them is the most suitable for cross calibration procedure and in what extent the electron beam energy will influence the result. All the dosimeters used here are certified by the SSDLs in 2-year period. The stability of calibration coefficient of thimble chambers is up to $0.2 \%$ in a 2 year-period, which means they follow the criterion ${ }^{19}$ of $0.3 \%$ long-term stability, which should be fulfilled by reference-class dosimeters.

The plane-parallel PPC05 chamber was the subject of the cross-calibration procedure. This Markus-type dosimeter is poorly recognized in the literature, including correction factors; therefore, the study could be of interest for worldwide users. Its basic characteristics in comparison with other frequently used plane-parallel detectors are presented in Table 1.

\subsection{Measurements in the reference medium}

Water phantom was used as the reference medium. Subsequently, for the cross-calibration procedure, a solid plate phantom made of RW3 material was also applied. Prior to the calibration procedure, the beam output (cGy/MU) of each electron beam was measured with the use of a plane-parallel ionization chamber PPC05. The result (the dose absorbed during emission of $100 \mathrm{MU}$ ) was calculated using the $\mathrm{N}_{\mathrm{d}, \mathrm{w}, \mathrm{Q} 0}$ coefficient taken from the SSDL certificate (established in ${ }^{60} \mathrm{Co}$ beam) and correction factors for: atmospheric conditions (air pressure and water temperature), ion recombination and polarizing voltage effects, and beam quality of: $\mathrm{k}_{\mathrm{p}, \mathrm{T}}, \mathrm{k}_{\mathrm{s}}, \mathrm{k}_{\mathrm{pol}}$ and $\mathrm{k}_{\mathrm{Q}, \mathrm{Q} 0}$, respectively were applied. Linac output of each electron beam was measured under reference conditions $\left(S S D=100 \mathrm{~cm}, 10 \times 10 \mathrm{~cm}^{2}\right.$ field size, $Z_{\text {ref }}$ depth), and in case the difference between the actual result and the reference value of $1 \mathrm{cGy} / \mathrm{MU}$ exceeds $0.5 \%$ - the efficiency of the beam was corrected to the reference level. The values of correction factors used for dose calculations based on the ionization (charge M) measured by the dosimetric setup for each electron beam are presented in Table 3. All three clinically used electron beams $(6,9$, and $15 \mathrm{MeV})$ were employed in the study, although the use of low-energy beams in the crosscalibration procedure is not recommended., ${ }^{\mathbf{1 , 2}}$ The reference point of the plane-parallel chamber was positioned at the measuring depth $\left(\mathrm{Z}_{\mathrm{ref}}\right)$, whereas for cylindrical chambers, the EPOM as an upstream shift by $0.5 r_{\text {cyl }}$ from the chamber central axis was applied according to the TRS-398 report. ${ }^{\mathbf{1}}$ These shifts were: $1.5 \mathrm{~mm}, 1.5 \mathrm{~mm}$, and $0.5 \mathrm{~mm}$ for FC65-G, CC13, and CC01 dosimeters, respectively. Subsequently, new measurements were obtained applying experimentally determined EPOM shifts (see subsection 2.4) for each dosimeter used.

All measurements were performed in the central beam axis for $10 \times 10 \mathrm{~cm}^{2}$ field size (electron applicator), since none of the beams is characterized by $\mathrm{R}_{50}>7 \mathrm{~g} / \mathrm{cm} .{ }^{21}$ The reproducing rate was maintained during all measurements at the level of $550-$ $600 \mathrm{MU} / \mathrm{min}$. The calibration was performed using the substitution method, i.e., dosimeters were placed in the measuring point within the phantom one after another. The accuracy of chambers positioning was $0.1 \mathrm{~mm}$ in water Blue Phantom2, whereas in the solid medium, it was limited to $1 \mathrm{~mm}$ of the thinnest SP34 phantom plate. 
Table 3. Correction factors used for dose determination on the base of charge collected by studied ionization chambers. The calibration coefficients $\mathrm{N}_{\mathrm{d}, \mathrm{w}, \mathrm{Q} 0}$ obtained by SSDLs in reference ${ }^{60} \mathrm{Co}$ beam together with the expanded uncertainties are also presented.

\begin{tabular}{|c|c|c|c|c|c|}
\hline Dosimeter & Electron beam nominal energy & $\mathbf{k}_{\mathbf{Q}, \mathbf{Q} \mathbf{0}}$ & $\mathbf{k}_{\mathrm{s}}$ & $\mathbf{k}_{\text {pol }}$ & $\mathbf{N}_{\mathrm{d}, \mathrm{w}, \mathbf{Q} 0}[\mathrm{cGy} / \mathrm{nC}]$ \\
\hline \multirow{3}{*}{ PPC05 S/N 792} & $6 \mathrm{MeV}$ & 0.934 & 1.004 & 0.994 & \multirow{3}{*}{$58.17 \pm 0.85$} \\
\hline & $9 \mathrm{MeV}$ & 0.923 & 1.003 & 0.996 & \\
\hline & $15 \mathrm{MeV}$ & 0.905 & 1.003 & 0.997 & \\
\hline \multirow{3}{*}{ FC65-G S/N 2684} & $6 \mathrm{MeV}$ & 0.924 & 1.008 & 0.998 & \multirow{3}{*}{$4.795 \pm 0.07$} \\
\hline & $9 \mathrm{MeV}$ & 0.921 & 1.008 & 1.000 & \\
\hline & $15 \mathrm{MeV}$ & 0.913 & 1.007 & 1.001 & \\
\hline \multirow{3}{*}{ CC13 S/N 12197} & $6 \mathrm{MeV}$ & 0.925 & 1.011 & 1.000 & \multirow{3}{*}{$26.55 \pm 0.27$} \\
\hline & $9 \mathrm{MeV}$ & 0.921 & 1.011 & 1.000 & \\
\hline & $15 \mathrm{MeV}$ & 0.913 & 1.011 & 1.000 & \\
\hline \multirow{3}{*}{ CC01 S/N 12185} & $6 \mathrm{MeV}$ & 0.950 & 1.004 & 0.980 & \multirow{3}{*}{$321.7 \pm 3.22$} \\
\hline & $9 \mathrm{MeV}$ & 0.944 & 1.005 & 0.988 & \\
\hline & $15 \mathrm{MeV}$ & 0.929 & 1.005 & 0.995 & \\
\hline
\end{tabular}

The charge collected by the chamber during the emission of $100 \mathrm{MU}$ was measured 10 times for each dosimeter in each condition, i.e., applying the voltage of $+300 \mathrm{~V}$ (normal operating voltage), $-300 \mathrm{~V}$ (required for polarization effect correction factor $\mathrm{k}_{\mathrm{pol}}$ ), and $+100 \mathrm{~V}$ (required for saturation/recombination effect correction factor $k_{s}$ ). Correction factors $k_{\text {pol }}$ and $k_{s}$ were obtained according to the TRS-398 recommendations. ${ }^{1}$ The measurement result of charge $\left(\mathrm{M}_{\mathrm{Q}}\right)$ for a given conditions setup was the arithmetic mean over 10 measurements, and the uncertainty was expressed as the standard deviation.

Correction factors used for dose calculation for each studied ionization chamber are presented in Table 3. TRS-398 report $^{1}$ provides values of beam quality correction factors $\mathrm{k}_{\mathrm{Q}, \mathrm{Q} 0}$ for cylindrical chambers starting from electron beam quality of $\mathrm{R}_{50}$ $\geq 4 \mathrm{~g} / \mathrm{cm}^{2}$ (in accordance with the recommendation not to use it for low-energy electron beam dosimetry). For the research purposes, $\mathrm{k}_{\mathrm{Q}, \mathrm{Q} 0}$ values for 6 and $9 \mathrm{MeV}$ beams were extrapolated using second-order polynomial fitting functions (Pearson's correlation factor of $\mathrm{R}=0.999$ for all chambers) to the data presented in table 7.III in TRS-398. ${ }^{1}$ Equations for FC65-G (1a), CC13 (1b) and CC01 (1c) ionization chambers, respectively were of the following forms:

$k_{Q, Q 0}=0.00003 \cdot\left(R_{50}\right)^{2}-0.00330 \cdot R_{50}+0.93197 \quad$ Eq. $1 \mathrm{a}$

$k_{Q, Q 0}=0.00003 \cdot\left(R_{50}\right)^{2}-0.00348 \cdot R_{50}+0.93309$ Eq. $1 \mathrm{~b}$

$k_{Q, Q 0}=0.00014 \cdot\left(R_{50}\right)^{2}-0.00717 \cdot R_{50}+0.96738$ Eq. $1 \mathrm{c}$

The beam quality correction factor of $\mathrm{k}_{\mathrm{Q}}$ for plane-parallel PPC05 ionization chamber was adopted from ${ }^{\mathbf{2 0}}$, since in TRS398 report $^{1}$ this dosimeter is omitted. It could be also found in IBA Dosimetry protocol ${ }^{22}$.

\subsection{Measurements in solid phantom}

Measurements were repeated in the solid phantom SP34 (IBA Dosimetry $\mathrm{GmbH}$ ) made of RW3 material. Although the TRS398 report $^{1}$ allows using plastic phantoms for low-energy beams $\left(\mathrm{R}_{50}<4 \mathrm{~g} / \mathrm{cm}^{3}\right)$, it was used for all studied beam quality in the presented study. The measuring depths of $\mathrm{Z}_{\mathrm{ref}}$ were rescaled according to TRS-398 guidelines (Equation 2):
$Z_{\text {ref,pl }}=\frac{Z_{\text {ref,water }}}{c_{p l}}\left[\frac{g}{\mathrm{~cm}^{2}}\right]=\frac{Z_{\text {ref,water }}}{c_{p l}} \cdot \rho_{p l}^{-1} \quad[\mathrm{~cm}] \quad$ Eq. 2

The set of depth-scaling factors $\mathrm{c}_{\mathrm{pl}}$ presented in ${ }^{\mathbf{1}}$ does not contain the density of RW3 phantom of $\rho_{\mathrm{pl}}=1.045 \mathrm{~g} / \mathrm{cm}^{3}$, but the values for lower and higher densities $\left(1.02-1.06 \mathrm{~g} / \mathrm{cm}^{3}\right)$ are present there. Therefore, the interpolation using a linear fitting function of: $c_{p l}=-0.749 \cdot \rho_{p l}+1.717(\mathrm{R}=0.982)$ was applied to obtain the value of $c_{\mathrm{pl}}=0.934$ for the $\rho_{\mathrm{pl}}=1.045 \mathrm{~g} / \mathrm{cm}^{3}$ density. The rescaled measuring depths for 6,9 and $15 \mathrm{MeV}$ electron beams were: $1.4 \mathrm{~cm}, 2.2 \mathrm{~cm}$ and $3.6 \mathrm{~cm}$, respectively. IPEM CoP reported value of $\mathrm{c}_{\mathrm{pl}}=0.98$ for clear polystyrene with quoted standard density of $1.045 \mathrm{~g} / \mathrm{cm}^{3}$ (the same as RW3 material). ${ }^{3}$ In that case, relevant measuring depths would be 1.4 $\mathrm{cm}, 2.1 \mathrm{~cm}$ and $3.4 \mathrm{~cm}$.

The electrometer readings $\left(\mathrm{M}_{\mathrm{Q}}\right)$ were also rescaled (Equation 3) following the TRS-398 guidelines, using the fluence correction factor $h_{p l}$. The value $h_{p l}=1.017$ for $\rho_{p l}=1.045$ $\mathrm{g} / \mathrm{cm}^{3}$ was similarly interpolated using linear fitting function of: $h_{p l}=0.345 \cdot \rho_{p l}+0.656(\mathrm{R}=0.875)$. IPEM CoP reported value of $h_{p l}=1.025$ for clear polystyrene. In that case, corrected electrometer readings would be $0.8 \%$ higher than used in the presented study. $^{3}$

$M_{Q}=M_{Q, p l} \cdot h_{p l}$

Eq. 3

The factors for rescaling the measuring depth and dose (ionization), discussed e.g. in $^{3}$ in more detail, arise from the differences in mechanisms of energy deposition between water and solid media.

\subsection{Determination of an effective point of measurement}

The effective point of measurement (EPOM) for plane-parallel chamber PPC05 has been determined by the comparison of percentage depth dose curve measured in RW3 solid phantom for $10 \times 10 \mathrm{~cm}^{2}$ field size and $15 \mathrm{MeV}$ beam with that obtained with EBT gafchromic films in the same conditions. EPOM was obtained as a shift of PDD curve to match the PDD measured with EBT film. The result of $1.6 \mathrm{~mm}$ found in the performed 
analysis is consistent with the value of $1.58 \mathrm{~mm}$ obtained $\mathrm{in}^{\mathbf{2 0}}$. Subsequently, PDD curves were measured in a water medium using each ionization chamber. Dosimeters were positioned according to their reference points, i.e., the centre of air cavity of thimble chambers and inner surface of entrance window of a plane-parallel chamber. EPOMs for thimble chambers were obtained as the shift of PDD curves to match that obtained for PPC05 with EPOM shifted by $1.6 \mathrm{~mm}$ downstream and averaged over three electron beam energies used in the presented study. In the text below, results obtained with EPOM shifted by the experimentally determined values are marked as (EPOM exp $)$.

\subsection{Cross-calibration procedure}

The cross-calibration of a plane-parallel ionization chamber has been conducted according to the IAEA TRS-398 formalism', based on the following equation:

$N_{d, w, Q \operatorname{cross}}=\frac{M_{Q \operatorname{cross}}^{r e f} \cdot k_{p, T} \cdot k_{s}^{r e f} \cdot k_{\text {pol }}^{r e f}}{M_{Q \operatorname{cross}} \cdot k_{p, T} \cdot k_{S} \cdot k_{p o l}} \cdot N_{d, w, Q 0}^{\text {ref }} \cdot k_{Q \operatorname{cross}, Q 0}^{\text {ref }} \quad$ Eq. 4

where: $\mathrm{N}_{\mathrm{d}, \mathrm{w}, \mathrm{Qcross}}$ is the cross-calibration coefficient. In the presented study, nine different values of this coefficient were determined using three electron beam energies and three cylindrical chambers as the reference dosimeters (superscript ref). $\mathrm{M}_{\mathrm{Q} \text { cross }}$ are averaged (here: over 10 subsequent measurements) electrometer readings, i.e. charge collected by each of ionization chamber, saturation and polarization correction factors $\mathrm{k}_{\mathrm{s}}$ and $\mathrm{k}_{\mathrm{pol}}$, respectively, are presented in Table 3, whereas $\mathrm{k}_{\mathrm{p}, \mathrm{T}}$ factor corrects the atmospheric conditions which might change between the measurements using different ionization chambers. Beam quality factors ( $\mathrm{k}_{\mathrm{Q} \text { cross, } \mathrm{Q} 0}$ ) for cylindrical chambers used as the reference once are presented in Table 3, since they are equal to $\mathrm{k}_{\mathrm{Q}, \mathrm{Q} 0}$ for ${ }^{60} \mathrm{Co}$ calibrated dosimeters.

\subsection{Dose reconstruction based on $\mathrm{N}_{\mathrm{d}, \mathrm{w}, \mathrm{Qcross}}$ coefficients}

From the clinical practice point of view, the precision of absolute dose assessment using $\mathrm{N}_{\mathrm{d}, \mathrm{w} \text {, Qcross }}$ coefficient seems to be more important than the difference between absolute values of $\mathrm{N}_{\mathrm{d}, \mathrm{w}, \mathrm{Q} 0}$ and $\mathrm{N}_{\mathrm{d}, \mathrm{w}, \mathrm{Qcross}}$ coefficients. Therefore, the calculation of dose deposited in a phantom medium by the electron beam of quality $\mathrm{Q}$ on the base of ionization $\left(\mathrm{M}_{\mathrm{Q}}\right)$ measured with the PPC05 dosimeter was made using the formula taken from TRS-398 report $^{1}$ in the form of Equation $5 \mathbf{a}$.

$D_{w, Q}=M_{Q} \cdot k_{p, T} \cdot k_{s} \cdot k_{\text {pol }} \cdot N_{d, w, Q c r o s s} \cdot k_{Q, Q c r o s s} \quad$ Eq. $5 \mathrm{a}$

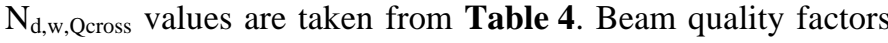
$\mathrm{k}_{\mathrm{Q}}$ in dosimetric reports, e.g. ${ }^{\mathbf{1 , 2}}$, are often shown only for a selected quality $Q_{\text {int }}=7.5 \mathrm{~g} / \mathrm{cm}^{2}$. Therefore, the Equation 5a should be modified as follows:

$D_{w, Q}=M_{Q} \cdot k_{p, T} \cdot k_{s} \cdot k_{p o l} \cdot N_{d, w, Q c r o s s} \cdot k_{Q, Q i n t} \cdot \frac{1}{k_{Q \text { int }, Q c r o s s}}$

Eq. $5 b$
The value of $\mathrm{k}_{\mathrm{Q}, \mathrm{Qin}}$ was taken for the pair of measured and $\mathrm{Q}_{\mathrm{int}}$ beams, whereas the value of $\mathrm{k}_{\text {Qint,Qcross }}$ was taken for the pair of $\mathrm{Q}_{\text {int }}$ and calibration beams. The most widely used reports TRS398 and TG-51 $1^{\mathbf{1 , 2}}$ do not contain data for PPC05 ionization chamber. Therefore, the values of $\mathrm{k}_{\mathrm{Q}}\left(\mathrm{R}_{50}\right)$ for the measured beam and $\mathrm{k}_{\mathrm{Qcross}}\left(\mathrm{R}_{50}\right)$ for the beam used for cross-calibration relative to the $\mathrm{Q}_{\text {int }}$ beam were obtained using the formula presented in $^{\mathbf{2 0}}$ with constant factors relevant for PPC05 dosimeter and described with Equation 6.

$k_{Q}=0.982+0.104 \cdot \exp \left[-\left(\frac{R_{50}}{4.248}\right)\right]$

Eq. 6

Values of $\mathrm{k}_{\mathrm{Q}}=0.934,0.923$ and 0.905 obtained with this method are in agreement with data contained in IBA report ${ }^{22}$ within $0.1 \%$.

\section{Results}

The cross-calibration coefficients $\mathrm{N}_{\mathrm{d}, \mathrm{w} \text {,Qcross }}$ obtained using various cylindrical reference chambers, electron beam energies, and measurement media are presented in Table 4. The uncertainty of these results is discussed in the subsection below. The values of the total uncertainty obtained here are in the range of $1.6 \%-2.9 \%$ and are higher compared to the expanded uncertainty of $\mathrm{N}_{\mathrm{d}, \mathrm{w}, \mathrm{Q} 0}$ coefficients given by SSDL $(\mathrm{U}=1.5 \%)$ for PPC05 ionization chamber. The $\mathrm{N}_{\mathrm{d}, \mathrm{w}, \mathrm{Q} \text { cross }}$ coefficients have lower uncertainty values in the case when reference cylindrical dosimeter has lower expanded uncertainty given by SSDL: $\mathrm{U}=$ $1.0 \%$ for $\mathrm{CC} 13$ chamber vs. $\mathrm{U}=1.5 \%$ for $\mathrm{FC} 65-\mathrm{G}$ chamber and the dosimeter readings have smaller standard deviation (have higher repeatability). Therefore, higher uncertainty for the results using $\mathrm{CC} 01$ dosimeter $(\mathrm{U}=1.0 \%)$ is connected with the higher variations among this chamber readings.

The PDD curves in water phantom measured using each studied ionization chamber are presented in Figure 1, together with the shifted results matching the EPOM positions presented in Table 5. The usage of EPOMs different than the recommended ones in IAEA TRS-398 resulted in changing the dosimeters' readings at $Z_{\text {ref }}$ depth of measurements up to $0.36 \%$. Therefore, the influence on the final $\mathrm{N}_{\mathrm{d}, \mathrm{w}, \mathrm{Qcross}}$ coefficients is from $-0.6 \%$ to $0.3 \%$.

The values of the ratio of cross calibration coefficient $\mathrm{N}_{\mathrm{d}, \mathrm{w}, \mathrm{Qcross}}$ with beam quality correction applied to ${ }^{60} \mathrm{Co} \mathrm{N}_{\mathrm{d}, \mathrm{w}, \mathrm{Q} 0}$ coefficient for PPC05 chamber are presented in Table 6. The average difference was $-1.5 \%$ whereas the median one was $1.1 \%$. Only in the case of $\mathrm{CC} 01$ reference dosimeter the obtained differences are larger than the expanded uncertainty given by $\operatorname{SSDL}(\mathrm{U}=1.5 \%)$. 


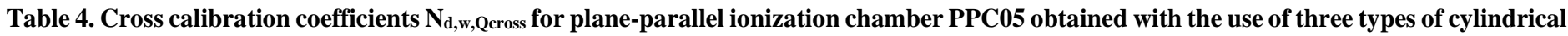
reference dosimeters in water and solid media.

\begin{tabular}{|c|c|c|c|c|}
\hline \multirow{2}{*}{\multicolumn{2}{|c|}{ Measurement conditions }} & \multicolumn{3}{|c|}{ Reference cylindrical ionization chamber } \\
\hline & & FC65-G & $\mathrm{CC13}$ & CC01 \\
\hline \multirow{3}{*}{ water phantom } & $6 \mathrm{MeV}$ beam & $53.50 \mathrm{cGy} / \mathrm{nC} \pm 2.0 \%$ & $53.87 \mathrm{cGy} / \mathrm{nC} \pm 1.6 \%$ & $53.26 \mathrm{cGy} / \mathrm{nC} \pm 2.4 \%$ \\
\hline & $9 \mathrm{MeV}$ beam & $53.06 \mathrm{cGy} / \mathrm{nC} \pm 2.0 \%$ & $53.44 \mathrm{cGy} / \mathrm{nC} \pm 1.7 \%$ & $52.93 \mathrm{cGy} / \mathrm{nC} \pm 2.1 \%$ \\
\hline & $15 \mathrm{MeV}$ beam & $52.36 \mathrm{cGy} / \mathrm{nC} \pm 2.0 \%$ & $52.71 \mathrm{cGy} / \mathrm{nC} \pm 1.7 \%$ & $52.11 \mathrm{cGy} / \mathrm{nC} \pm 2.9 \%$ \\
\hline \multirow{3}{*}{$\begin{array}{l}\text { water phantom } \\
\left(\mathrm{EPOM}_{\exp }\right)\end{array}$} & $6 \mathrm{MeV}$ beam & $53.61 \mathrm{cGy} / \mathrm{nC} \pm 2.0 \%$ & $54.03 \mathrm{cGy} / \mathrm{nC} \pm 1.6 \%$ & $53.26 \mathrm{cGy} / \mathrm{nC} \pm 2.4 \%$ \\
\hline & $9 \mathrm{MeV}$ beam & $53.06 \mathrm{cGy} / \mathrm{nC} \pm 2.0 \%$ & $53.47 \mathrm{cGy} / \mathrm{nC} \pm 1.7 \%$ & $52.93 \mathrm{cGy} / \mathrm{nC} \pm 2.1 \%$ \\
\hline & $15 \mathrm{MeV}$ beam & $52.09 \mathrm{cGy} / \mathrm{nC} \pm 2.0 \%$ & $52.38 \mathrm{cGy} / \mathrm{nC} \pm 1.7 \%$ & $51.92 \mathrm{cGy} / \mathrm{nC} \pm 2.9 \%$ \\
\hline \multirow{3}{*}{$\begin{array}{l}\text { solid phantom } \\
\text { RW3 }\end{array}$} & $6 \mathrm{MeV}$ beam & $52.97 \mathrm{cGy} / \mathrm{nC} \pm 2.0 \%$ & $53.17 \mathrm{cGy} / \mathrm{nC} \pm 1.6 \%$ & $53.44 \mathrm{cGy} / \mathrm{nC} \pm 2.4 \%$ \\
\hline & $9 \mathrm{MeV}$ beam & $52.94 \mathrm{cGy} / \mathrm{nC} \pm 2.0 \%$ & $53.93 \mathrm{cGy} / \mathrm{nC} \pm 1.7 \%$ & $53.52 \mathrm{cGy} / \mathrm{nC} \pm 2.1 \%$ \\
\hline & $15 \mathrm{MeV}$ beam & $52.59 \mathrm{cGy} / \mathrm{nC} \pm 2.0 \%$ & $53.24 \mathrm{cGy} / \mathrm{nC} \pm 1.7 \%$ & $53.26 \mathrm{cGy} / \mathrm{nC} \pm 2.9 \%$ \\
\hline
\end{tabular}

Table 5. Values of EPOM shifts determined experimentally and applied during the analysis of their influence on cross calibration coefficients in comparison with literature data.

\begin{tabular}{|c|c|c|c|c|}
\hline \multirow{3}{*}{ Ionization chamber } & \multicolumn{4}{|c|}{ EPOM } \\
\hline & \multicolumn{2}{|c|}{ experiment } & \multicolumn{2}{|c|}{ literature } \\
\hline & Absolute value & Fraction of $R_{\text {cav }}$ & Absolute value & Fraction of $R_{\text {cav }}$ \\
\hline PPC05 & $1.6 \mathrm{~mm}$ downstream & - & $1.58 \mathrm{~mm}^{20}$ & - \\
\hline FC 65-G & $1.0 \mathrm{~mm}$ upstream & 0.32 & $1.09 \mathrm{~mm}^{20}$ & 0.35 \\
\hline $\mathrm{CC} 13$ & $1.2 \mathrm{~mm}$ upstream & 0.4 & $0.7 \mathrm{~mm}^{\mathbf{2 0}}$ & 0.23 \\
\hline $\mathrm{CC} 01$ & $0.5 \mathrm{~mm}$ upstream & 0.5 & - & - \\
\hline
\end{tabular}

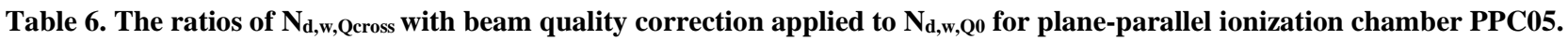

\begin{tabular}{|c|c|c|c|c|}
\hline \multirow{2}{*}{ Measurement conditions } & & \multicolumn{3}{|c|}{ Reference cylindrical ionization chamber } \\
\hline & & FC65-G & $\mathrm{CC13}$ & $\mathrm{CC01}$ \\
\hline \multirow{3}{*}{ water phantom } & $6 \mathrm{MeV}$ beam & 0.996 & 0.994 & 0.957 \\
\hline & $9 \mathrm{MeV}$ beam & 0.991 & 0.997 & 0.964 \\
\hline & $15 \mathrm{MeV}$ beam & 0.986 & 0.992 & 0.964 \\
\hline \multirow{3}{*}{ water phantom $\left(\mathrm{EPOM}_{\mathrm{exp}}\right)$} & $6 \mathrm{MeV}$ beam & 0.997 & 0.994 & 0.957 \\
\hline & $9 \mathrm{MeV}$ beam & 0.991 & 0.998 & 0.964 \\
\hline & $15 \mathrm{MeV}$ beam & 0.981 & 0.986 & 0.961 \\
\hline \multirow{3}{*}{ solid phantom RW3 } & $6 \mathrm{MeV}$ beam & 0.986 & 0.989 & 0.967 \\
\hline & $9 \mathrm{MeV}$ beam & 0.988 & 1.006 & 0.975 \\
\hline & $15 \mathrm{MeV}$ beam & 0.990 & 1.003 & 0.985 \\
\hline
\end{tabular}

\subsection{Uncertainty discussion}

The combined standard uncertainty of $\mathrm{N}_{\mathrm{d}, \mathrm{w}, \mathrm{Qcross}}$ values were determined using a logarithmic derivative method, since this coefficient is calculated based on Equation 4 as the product of quantities having their own uncertainty stated. It has been assumed that the components of Equation 4 are independent quantities. Therefore, the final relative uncertainty

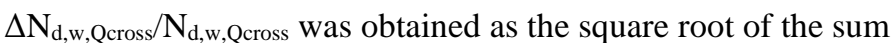
of component relative uncertainties in quadrature. Uncertainties of $\Delta \mathrm{k}_{\mathrm{s}}$ and $\Delta \mathrm{k}_{\mathrm{pol}}$, calculated using the complete differential method, were in the range of $0.1-2.0 \%$, depending on the electron beam energy and reference cylindrical dosimeter used. Standard deviation from 10 subsequent measurements was adopted as the uncertainty of charge $\mathrm{M}$ collected by each of the ionization chambers. It ranges from $0.03 \%$ to $0.6 \%$. The uncertainty of $\Delta \mathrm{N}_{\mathrm{d}, \mathrm{w}, \mathrm{Q} 0}$ of cylindrical ionization chambers is the expanded uncertainty (U) given in the SSDL calibration certificate. For compact chambers $\mathrm{CC} 13$ and $\mathrm{CC} 01$ it was $1.0 \%$, whereas for the Farmer type chamber, it was $1.5 \%$. The uncertainty of beam quality correction factor $\Delta \mathrm{k}_{\mathrm{Q}}$ was assessed based on the discussion presented $i^{1}$ at the level of $1.225 \%$, taking into account the uncertainties of: mass stopping powers, $\mathrm{W}_{\text {air }}$ values and perturbation correction factors for cylindrical ionization chambers.

\subsection{Testing the correctness of cross-calibration}

The data published by the Polish $\mathrm{SSDL}^{8}$ shows no differences between $\mathrm{N}_{\mathrm{d} \text {,w }}$ calibration coefficients obtained in the reference ${ }^{60} \mathrm{Co}$ beam and in the high-energy $(22 \mathrm{MeV})$ electron beam. Therefore, to test the correctness of cross-calibration, which was carried out in the clinical conditions, one may compare the values of $\mathrm{N}_{\mathrm{d}, \mathrm{w}, \mathrm{Q} 0}$ (SSDL certificate) and $\mathrm{N}_{\mathrm{d}, \mathrm{w}, \mathrm{Q} \text { cross }}$ (clinical electron beam) directly, with beam quality correction applied when obtained in beams having an energy below recommended one, or compare the doses calculated using the appropriate equation, e.g. given in TRS-398 report, as in presented study. 
The precision of performed cross-calibration would be then the difference between the dose based on $\mathrm{N}_{\mathrm{d}, \mathrm{w}, \mathrm{Q} 0}$ (assuming this is a true value) and the dose resulted from Equations 5a and $\mathbf{5 b}$.

\section{Measurements in water}

The absolute values of $\mathrm{N}_{\mathrm{d}, \mathrm{w}, \mathrm{Qcross}}$ obtained in a reference medium, presented in Table 4, are 7\% - 10\% lower than the $\mathrm{N}_{\mathrm{d}, \mathrm{w}, \mathrm{Q} 0}$ value from the PPC05 chamber certificate (Table 3). Taking into account the beam quality correction factors $\mathrm{k}_{\mathrm{Q}, \mathrm{Q} 0}$ (Table 3 ) for all cylindrical chambers used $\mathrm{N}_{\mathrm{d}, \mathrm{w} \text {,Qcross }}$ values (Table 4) were rescaled to the range of $55.70 \mathrm{cGy} / \mathrm{nC}-58.01 \mathrm{cGy} / \mathrm{nC}$, i.e. they are lower than the value $\mathrm{N}_{\mathrm{d}, \mathrm{w}, \mathrm{Q} 0}=58.17 \mathrm{cGy} / \mathrm{nC}$ (Table 3) stated by the SSDL using ${ }^{60} \mathrm{Co}$ beam by up to $1.5 \%$ for $\mathrm{FC}-65 \mathrm{G}$, up to $0.8 \%$ for $\mathrm{CC} 13$ and up to $4.3 \%$ for CC01 dosimeters, depending on the electron beam energy.

The results of electron beam output [cGy/MU] calculated on the base of $\mathrm{N}_{\mathrm{d}, \mathrm{w}, \mathrm{Qcross}}$ using Equation 5b are presented in Table 7. The measurements were performed in the reference conditions. Therefore, the obtained values should be $1 \mathrm{cGy} / \mathrm{MU}$ $\pm 1.5 \%$. At the time of performed measurements, the efficiencies determined on the base of $\mathrm{N}_{\mathrm{d}, \mathrm{w}, \mathrm{Q} 0}\left({ }^{60} \mathrm{Co}\right.$ beam) were the following: $1.004 \mathrm{cGy} / \mathrm{MU}$ for $6 \mathrm{MeV}$ beam, $1.003 \mathrm{cGy} / \mathrm{MU}$ for $9 \mathrm{MeV}$ beam, and $0.997 \mathrm{cGy} / \mathrm{MU}$ for $15 \mathrm{MeV}$ beam. The most precise results were obtained in the case when $\mathrm{CC} 13$ dosimeter was used for the determination of $\mathrm{N}_{\mathrm{d}, \mathrm{w}, \mathrm{Qcross}}$ regardless of the beam energy used for calibration purposes. The higher beam energy, the more precise results were got using $\mathrm{N}_{\mathrm{d} \text {,w, Qcross }}$ obtained with farmer type chamber.

\section{Measurements in a solid phantom}

The absolute values of $\mathrm{N}_{\mathrm{d}, \mathrm{w} \text {, Qcross }}$ in a solid phantom are also $7 \%$ - $10 \%$ lower than the $\mathrm{N}_{\mathrm{d}, \mathrm{w}, \mathrm{Q} 0}$ value from the PPC05 chamber certificate (see: Tables 3 and 4). Taking into account the beam quality correction factors $\mathrm{k}_{\mathrm{Q}, \mathrm{Q} 0}$ (Table 3) for all cylindrical chambers used $\mathrm{N}_{\mathrm{d}, \mathrm{w}, \mathrm{Qcross}}$ values (Table 4) were rescaled to the range of $56.24 \mathrm{cGy} / \mathrm{nC}-58.53 \mathrm{cGy} / \mathrm{nC}$, i.e. they differ from the value $\mathrm{N}_{\mathrm{d}, \mathrm{w}, \mathrm{Q} 0}=58.17 \mathrm{cGy} / \mathrm{nC}$ (Table 3) stated by the SSDL using ${ }^{60} \mathrm{Co}$ beam from -1.0 to $-1.4 \%$ for $\mathrm{FC}-65 \mathrm{G}$, from $-1.1 \%$ to $0.3 \%$ for $\mathrm{CC} 13$ and from $-1.5 \%$ to $-3.3 \%$ for $\mathrm{CC} 01$ dosimeters, depending on the electron beam energy.

The results of electron beam output [cGy/MU] calculated on the base of $\mathrm{N}_{\mathrm{d}, \mathrm{w}, \text { Qcross }}$ using Equation 5b are presented in Table 8. The most precise results were obtained on the base of $\mathrm{N}_{\mathrm{d}, \mathrm{w}, \text { Qcross }}$ determined with the use of various reference dosimeters for various beam energies used for calibration purposes.

Table 7. Electron beam output [cGy/MU] obtained using cross calibration coefficients $\mathbf{N}_{\mathrm{d}, \mathrm{w}, \mathrm{Q} \text {, }}$ (coss determined in water phantom on the base of ionization measured in water medium with the use of PPC05 dosimeter. The most precise results are marked bold. Values in parentheses are the percentage deviations from the actual beam efficiencies.

\begin{tabular}{|c|c|c|c|c|}
\hline \multirow{2}{*}{$\begin{array}{l}\text { Reference dosimeter } \\
\text { in cross calibration procedure }\end{array}$} & \multirow{2}{*}{ Beam used for cross calibration } & \multicolumn{3}{|c|}{ Measured beam } \\
\hline & & $6 \mathrm{MeV}$ & $9 \mathrm{MeV}$ & $15 \mathrm{MeV}$ \\
\hline FC65-G & & $0.989(-1.5 \%)$ & $0.988(-1.5 \%)$ & $0.981(-1.5 \%)$ \\
\hline CC13 & $6 \mathrm{MeV}$ & $0.996(-0.9 \%)$ & $0.995(-0.9 \%)$ & $\mathbf{0 . 9 8 8}(-0.9 \%)$ \\
\hline $\mathrm{CC} 01$ & & $0.984(-2.0 \%)$ & $0.983(-2.0 \%)$ & $0.977(-2.0 \%)$ \\
\hline FC65-G & & $0.992(-1.2 \%)$ & $0.991(-1.2 \%)$ & $0.985(-1.2 \%)$ \\
\hline $\mathrm{CC} 13$ & $9 \mathrm{MeV}$ & $0.999(-0.5 \%)$ & $0.999(-0.5 \%)$ & $\mathbf{0 . 9 9 2}(-0.5 \%)$ \\
\hline $\mathrm{CC} 01$ & & $0.990(-1.4 \%)$ & $0.989(-1.4 \%)$ & $0.982(-1.4 \%)$ \\
\hline FC65-G & & $0.999(-0.5 \%)$ & $0.998(-0.5 \%)$ & $0.991(-0.5 \%)$ \\
\hline CC13 & $15 \mathrm{MeV}$ & $\mathbf{1 . 0 0 5}(0.1 \%)$ & $1.005(0.1 \%)$ & $\mathbf{0 . 9 9 8}(0.1 \%)$ \\
\hline $\mathrm{CC} 01$ & & $0.994(-1.0 \%)$ & $0.993(-1.0 \%)$ & $0.987(-1.0 \%)$ \\
\hline
\end{tabular}

Table 8. Electron beam output [cGy/MU] obtained using cross calibration coefficients $\mathbf{N}_{\mathrm{d}, \mathrm{w}, \mathrm{Qcross}}$ determined in solid SP34 phantom on the base of ionization measured in RW3 medium with the use of PPC05 dosimeter. The most precise results are marked bold. Values in parentheses are the percentage deviations from the actual beam efficiencies.

\begin{tabular}{|c|c|c|c|c|}
\hline \multirow{2}{*}{$\begin{array}{l}\text { Reference dosimeter in cross } \\
\text { calibration procedure }\end{array}$} & \multirow{2}{*}{ Beam used for cross calibration } & \multicolumn{3}{|c|}{ Measured beam } \\
\hline & & $6 \mathrm{MeV}$ & $9 \mathrm{MeV}$ & $15 \mathrm{MeV}$ \\
\hline FC65-G & & $0.980(-2.4 \%)$ & $0.975(-2.8 \%)$ & $0.964(-3.2 \%)$ \\
\hline $\mathrm{CC} 13$ & $6 \mathrm{MeV}$ & $0.984(-2.1 \%)$ & $0.979(-2.5 \%)$ & $0.968(-2.9 \%)$ \\
\hline $\mathrm{CC} 01$ & & $\mathbf{0 . 9 8 9}(-1.5 \%)$ & $\mathbf{0 . 9 8 4}(-2.0 \%)$ & $\mathbf{0 . 9 7 3}(-2.4 \%)$ \\
\hline FC65-G & & $0.991(-1.3 \%)$ & $0.986(-1.7 \%)$ & $0.975(-2.1 \%)$ \\
\hline $\mathrm{CC} 13$ & $9 \mathrm{MeV}$ & $1.009(0.5 \%)$ & $1.004(0.1 \%)$ & $\mathbf{0 . 9 9 3}(-0.3 \%)$ \\
\hline $\mathrm{CC} 01$ & & $\mathbf{1 . 0 0 2}(-0.3 \%)$ & $0.997(-0.7 \%)$ & $0.986(-1.1 \%)$ \\
\hline FC65-G & & $1.004(-0.01 \%)$ & $\mathbf{0 . 9 9 9}(-0.4 \%)$ & $0.988(-0.8 \%)$ \\
\hline CC13 & $15 \mathrm{MeV}$ & $1.017(1.2 \%)$ & $1.012(0.8 \%)$ & $1.001(0.4 \%)$ \\
\hline $\mathrm{CC} 01$ & & $1.017(1.3 \%)$ & $1.012(0.8 \%)$ & $\mathbf{1 . 0 0 1}(0.4 \%)$ \\
\hline
\end{tabular}


Table 9. Electron beam output [cGy/MU] obtained using cross calibration coefficients $\mathbf{N}_{\mathrm{d}, \mathrm{w}, \mathrm{Qcross}}$ determined in water phantom on the base of ionization measured in water medium with the use of PPC05 dosimeter. Measurements were performed with applying experimentally determined EPOMs for all chambers used. The most precise results are marked bold. Values in parentheses are the percentage deviations from the actual beam efficiencies.

\begin{tabular}{|c|c|c|c|c|c|}
\hline \multirow{2}{*}{$\begin{array}{l}\text { Reference } \\
\text { dosimeter }\end{array}$} & \multirow{2}{*}{$\mathbf{E P O M}_{\exp }[\mathrm{mm}]$} & \multirow{2}{*}{$\begin{array}{l}\text { Beam used for cross } \\
\text { calibration }\end{array}$} & \multicolumn{3}{|c|}{ Measured beam } \\
\hline & & & $6 \mathrm{MeV}$ & $9 \mathrm{MeV}$ & $15 \mathrm{MeV}$ \\
\hline FC65-G & 1.0 & \multirow{3}{*}{$6 \mathrm{MeV}$} & $0.991(-1.3 \%)$ & $0.990(-1.3 \%)$ & $0.984(-1.3 \%)$ \\
\hline CC13 & $0.7,1.2$ & & $0.999(-0.6 \%)$ & $0.998(-0.6 \%)$ & $0.991(-0.6 \%)$ \\
\hline $\mathrm{CC} 01$ & 0.5 & & $0.984(-2.0 \%)$ & $0.983(-2.0 \%)$ & $0.980(-1.7 \%)$ \\
\hline FC65-G & 1.0 & \multirow{3}{*}{$9 \mathrm{MeV}$} & $0.992(-1.2 \%)$ & $0.991(-1.2 \%)$ & $0.988(-0.9 \%)$ \\
\hline CC13 & $0.7,1.2$ & & $1.000(-0.4 \%)$ & $0.999(-0.4 \%)$ & $0.996(-0.1 \%)$ \\
\hline $\mathrm{CC} 01$ & 0.5 & & $0.990(-1.4 \%)$ & $0.989(-1.4 \%)$ & $0.986(-1.1 \%)$ \\
\hline FC65-G & 1.0 & \multirow{4}{*}{$15 \mathrm{MeV}$} & $0.994(-1.1 \%)$ & $0.993(-1.1 \%)$ & $0.990(-0.7 \%)$ \\
\hline $\mathrm{CC} 13$ & 0.7 & & $0.999(-0.5 \%)$ & $0.998(-0.5 \%)$ & $0.995(-0.2 \%)$ \\
\hline $\mathrm{CC} 13$ & 1.2 & & $1.001(-0.3 \%)$ & $1.001(-0.3 \%)$ & $0.997(0.0 \%)$ \\
\hline $\mathrm{CC} 01$ & 0.5 & & $0.990(-1.4 \%)$ & $0.990(-1.4 \%)$ & $0.987(-1.0 \%)$ \\
\hline
\end{tabular}

\section{Measurements using experimental EPOM}

The use of an experimentally determined effective point of measurement for each dosimeter involved in the presented study resulted in the correction of $\mathrm{N}_{\mathrm{d}, \mathrm{w}, \mathrm{Qcross}}$ values obtained in reference medium with EPOM taken from TRS-398 up to $0.3 \%$ for $6 \mathrm{MeV}, 0.1 \%$ for $9 \mathrm{MeV}$ and from $-0.6 \%$ to $-0.4 \%$ for 15 $\mathrm{MeV}$ beams depending on reference dosimeter used.

The results of electron beam output [cGy/MU] calculated on the base of $\mathrm{N}_{\mathrm{d}, \mathrm{w}, \mathrm{Q} \text {, ross }}$ using Equation $\mathbf{5 b}$ are presented in Table 9 for water phantom with experimentally determined EPOM shift applied. Comparing with data presented in Table 7, the deviations from the actual beam outputs were decreased up to $0.4 \%$ and up to if $6 \mathrm{MeV}$ and $9 \mathrm{MeV}$ beam were used for cross-calibration. In the case of $15 \mathrm{MeV}$ beam usage for $\mathrm{N}_{\mathrm{d}, \mathrm{w}, \text { Qcross }}$ estimation, the precision of subsequent dose reconstruction decreases up to $0.5 \%$.

\section{Discussion}

According to the AAPM guidelines ${ }^{2}$, the use of cylindrical ionization chambers is possible even for $6 \mathrm{MeV}$ electron beams, although the recommended threshold is $10 \mathrm{MeV}$. It has been shown ${ }^{6}$ that the differences for a given type of cylindrical chamber between low- and high-energy electron beams are of the order of $\sim 0.4 \%$. The use of a Farmer type FC65-G dosimeter introduces the difference between $18 \mathrm{MeV}$ and $4 \mathrm{MeV}$ electron beams of the order of $0.2 \% .^{6}$ Nevertheless, the data in Table 7 show the difference in dose reconstruction of the order of $1 \%$ when the plane-parallel chamber was calibrated in low-energy (6 MeV) and high-energy (15 MeV) electron beam using all studied thimble chambers. The differences decreased up to a maximum of $0.7 \%$, when more relevant (chamber-specific) effective points of measurement were applied during the crosscalibration procedure, which could be seen in Table 9. In studied conditions, differences in dose reconstruction when applying various EPOMs (here: $1.2 \mathrm{~mm}$ established from performed measurements vs. $0.7 \mathrm{~mm}$ taken from literature) for CC13 chamber are $0.2 \%$. However, one could expect higher discrepancies for $\mathrm{N}_{\mathrm{d}, \mathrm{w}, \text { Qcross }}$ obtained in electron beams with higher energies, provided the measurements are performed at $Z_{\text {ref }}$ depth. Due to increased distance between $D_{\max }$ and $Z_{\text {ref }}$ depths, even minimal shift introduces a not negligible change in dosimeter reading since for high-energy electron beams the point of $Z_{\text {ref }}$ is on the slope of PDD curve (Figure 1).

The comparison of absorbed dose to water measured according to different dosimetric protocols is widely presented $\mathrm{in}^{21}$. The results in the case of using a plane-parallel chambers cross calibrated against Farmer type chamber, which had an $\mathrm{N}_{\mathrm{d}, \mathrm{w}, \mathrm{Q} 0}$ determined in ${ }^{60} \mathrm{Co}$, differ by $0.3 \%-0.7 \%$ for Markus, Roos, and NACP dosimeters when TRS-398 and TG-51 protocols are used. In the case when absorbed dose in electron beam is measured using cylindrical and Ross chambers calibrated in ${ }^{60} \mathrm{Co}$ reference beam, application of DIN 6800-2 protocol introduces the differences of $0.15 \%-0.3 \%$ compared to the dose determined according to TRS-398 protocol, whereas TG-51 introduces the differences of $0.6 \%-0.8 \%$ for planeparallel dosimeters and $0.2 \%-0.6 \%$ for cylindrical chambers. Detailed analysis of dose discrepancies depending on the protocol used, presented in $^{\mathbf{2 3}}$, shows the differences between TRS-398 and DIN 6800-2 protocols at the level of 0.1\%.

There are some protocols, e.g ${ }^{24}$, which advice using the same beam quality for cross-calibration that is subsequently measured by plane-parallel ionization chamber. This allows the lowenergy electron beam to be used if only it is clinically used. However, obtained data presented in Table 7 shows that dose measured in each studied electron beam is more precise reconstructed when $\mathrm{N}_{\mathrm{d} \text {,w, Qcross }}$ obtained in $15 \mathrm{MeV}$ beam is used regardless of the thimble chamber used as a reference one.

The issue of EPOM localization in phantoms should also be considered. As shown in ${ }^{\mathbf{1 0}}$, the reference point displacement reaches $1.51 \mathrm{~mm}, 1.19 \mathrm{~mm}, 0.36 \mathrm{~mm}$, and $-0.2 \mathrm{~mm}$ for FC 65G, CC13, CC01, and PPC05 chambers, respectively, whereas according to TRS-398 guidelines, the shift of $1.5 \mathrm{~mm}, 1.5 \mathrm{~mm}$, $0.5 \mathrm{~mm}$ and $0.0 \mathrm{~mm}$ was applied for these dosimeters, respectively. Since the obtained results of $\mathrm{N}_{\mathrm{d}, \mathrm{w}, \text { Qcross }}$ coefficient seem to be in most cases undervalued, taking into account 
Equation 4, it could be concluded that the EPOM mismatch influences more on the thimble chambers readings $\left(\mathrm{M}_{\mathrm{Q}, \text { ref }}\right)$ than on plane-parallel chamber indications in terms of underestimating the $\mathrm{M}_{\mathrm{Q}}$ readings. If the $\mathrm{N}_{\mathrm{d} \text {,w, Qcross }}$ values were only EPOM dependent, the usage of Farmer-type chamber should give the most precise results, or even overestimated ones. Since the correction factors $\mathrm{k}_{\mathrm{Q}}, \mathrm{k}_{\mathrm{S}}$ and $\mathrm{k}_{\mathrm{pol}}$ have to be also taken into account in the procedure of absolute dose measurements, the denominator in Equation 4 is high enough in comparison with the rest of the factors to produce the undervalued $\mathrm{N}_{\mathrm{d}, \mathrm{w}, \mathrm{Qcross}}$ coefficient in case of FC 65-G chamber as well. Experimentally determined shifts of EPOM show that $0.5 \mathrm{r}_{\text {cav }}$ recommended in TRS-398 for thimble chambers is the maximal that should be used, whereas the additional shift of EPOM for plane-parallel PPC05 chamber of $0.6 \mathrm{~mm}$ should be considered. The systematic error introduced by incorrect EPOM shift usage seems to be more crucial when a high-energy beam is used for cross-calibration. As presented in Figure 1, when measurements are performed at $\mathrm{Z}_{\mathrm{ref}}$ depth, the higher the electron beam energy, the higher discrepancies in PDD curves. If one follows the new concept of calibration ${ }^{25}$, the discrepancies could be maintained at the level of $0.5 \%$.

\section{Conclusions}

The use of $15 \mathrm{MeV}$ electron beam for cross-calibration purposes, although the recommendations are for at least $16 \mathrm{MeV}^{\mathbf{1 8}}$, enables to obtain in water medium an $\mathrm{N}_{\mathrm{d}, \mathrm{w} \text {,Qcross }}$ coefficient, which reproduces the absolute dose in water with the precision of the order of $0.1 \%$ and in solid phantom between $-0.8 \%$ and $0.4 \%$ compared to the case when $\mathrm{N}_{\mathrm{d}, \mathrm{w}, \mathrm{Q} 0}$ obtained in ${ }^{60} \mathrm{Co}$ beam is used for the dose determination.

Attention must be paid to the choice of reference cylindrical dosimeter. End-user for cross-calibration purposes in electron beams with $\mathrm{E} \leq 15 \mathrm{MeV}$ is advised to choose the reference dosimeter of the smallest active volume and with electrodes made of water-equivalent material. Among three tested cylindrical ionization chambers (FC65-G, CC13, and CC01) as potential reference dosimeters for the cross-calibration procedure, the most precise results of $\mathrm{N}_{\mathrm{d}, \mathrm{w}, \mathrm{Qcross}}$ coefficients, and absolute dose reconstruction were obtained using compact chamber $\mathrm{CC} 13$. This dosimeter has a relatively small active volume (in comparison with FC65-G chamber) and waterequivalent material of inner electrode (while CC01 chamber has not). The results presented in Table 7 indicate that the precision of dose assessment based of $\mathrm{N}_{\mathrm{d}, \mathrm{w}, \mathrm{Qcross}}$ coefficients obtained in all studied electron beams using FC65-G dosimeter do not exceed the expanded uncertainty $(U=1.5 \%)$ for this ionization chamber stated by SSDL. The presented study has shown that the cross-calibration coefficient obtained in a low-energy (6 $\mathrm{MeV}$ ) electron beam tends to underestimate the absolute dose values of $0.5 \%-2 \%$ depending on the choice of reference cylindrical dosimeter.

(a)

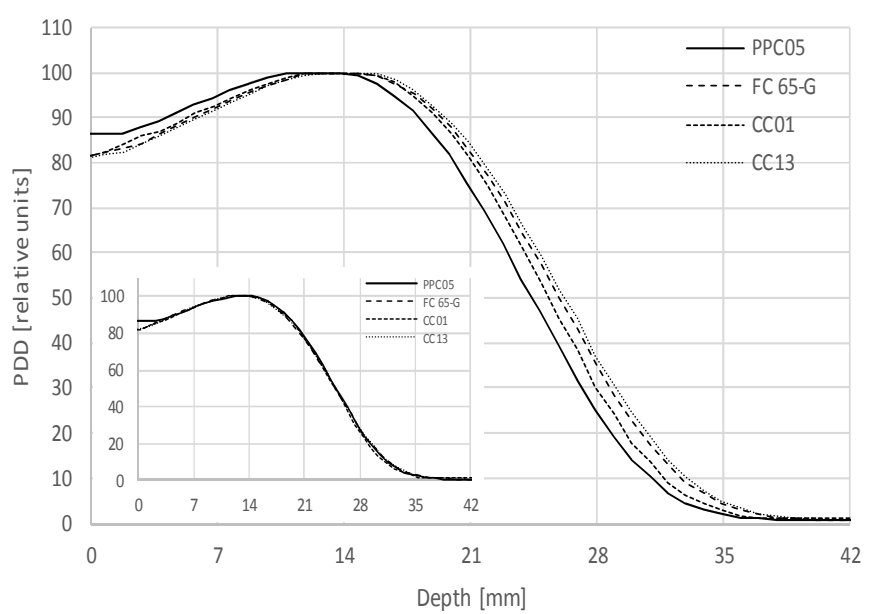

(b)

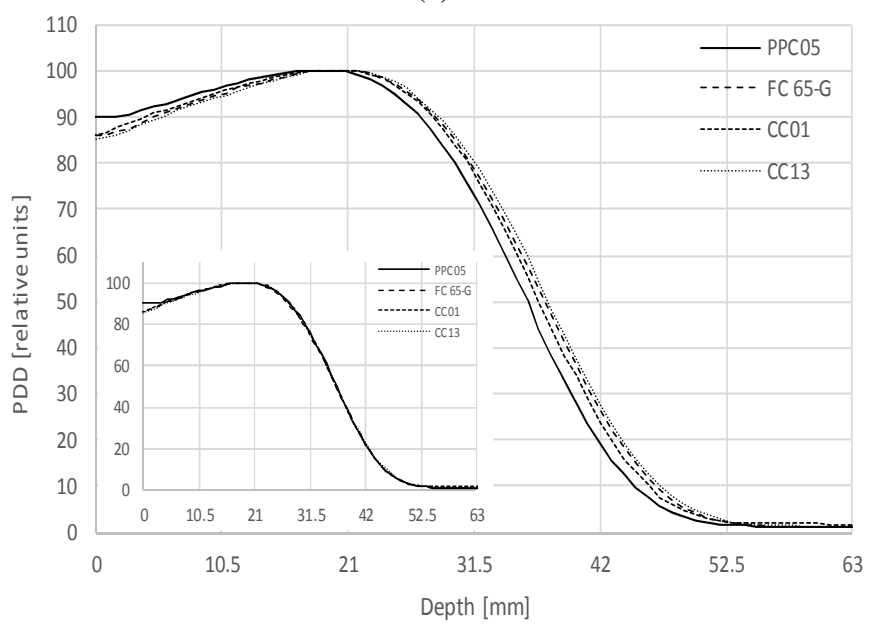

(c)

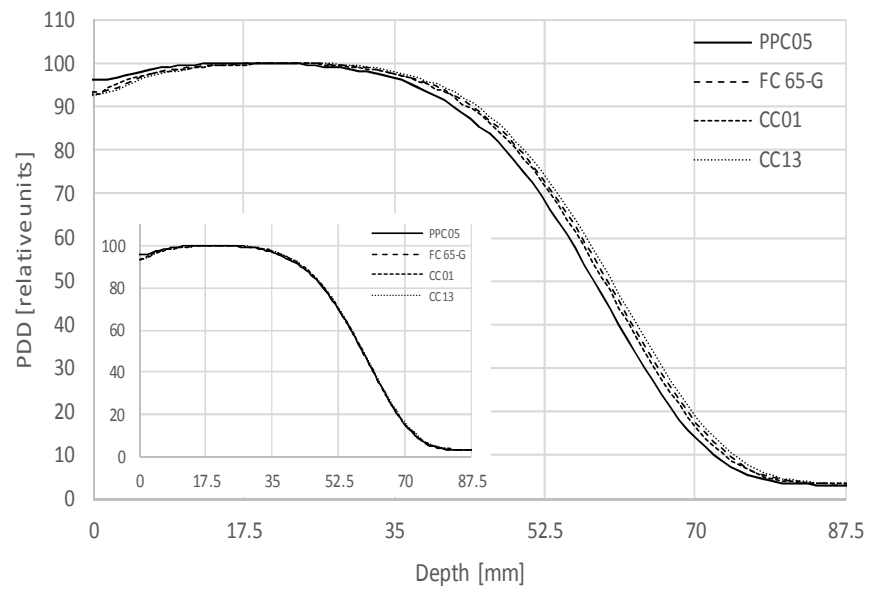

Figure 1. PDD curves measured with all studied ionization chambers without applying correction for effective point of measurement, i.e. the starting point (zero position) was set in the centre of thimble chambers and at the inner surface of planeparallel chamber, i.e. at the reference points provided by the manufacturer. The differences are clearly seen at depths greater

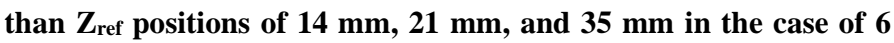
$\mathrm{MeV}$ (a), $9 \mathrm{MeV}$ (b), and $15 \mathrm{MeV}$ (c) beams, respectively. The insets show PDD curves after the EPOM shifts according to the data presented in Table 5. 
In the case when Farmer type FC65-G dosimeter was used, the $\mathrm{N}_{\mathrm{d}, \mathrm{w}, \mathrm{Qcross}}$ values were systematically lowered (from -0.4 to $1.4 \%$ ), and this effect is more pronounced in solid phantom than

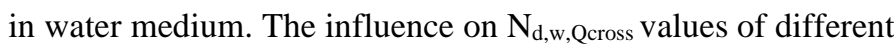
positions of EPOM than recommended in CoPs is more pronounced for beams with higher energies, i.e., when localization of $\mathrm{Z}_{\text {ref }}$ considerably differs from the depth of dose maximum (as could be seen in Figure 1). Therefore, if the exact position of EPOM could not be determined by the user, lower energy of electron beam should be considered for the crosscalibration procedure. The precision of $\mathrm{N}_{\mathrm{d}, \mathrm{w}, \mathrm{Qcross}}$ compared to the $\mathrm{N}_{\mathrm{d}, \mathrm{w}, \mathrm{Q} 0}$ increases with increasing electron beam energy used for the cross-calibration procedure. For $\mathrm{CC} 01$ ionization chamber, the discrepancies decreased from $-2 \%$ to $-1 \%$ and for $\mathrm{CC} 13$ dosimeter from $-0.9 \%$ to $0.1 \%$ when $6 \mathrm{MeV}$ beam was replaced by $15 \mathrm{MeV}$ beam. The overall differences between 6 $\mathrm{MeV}$ and $15 \mathrm{MeV}$ electron beams are of the order of $1 \%$ regardless of cylindrical dosimeter used. However, minimal and maximal values of $\mathrm{N}_{\mathrm{d}, \mathrm{w} \text {, Qcross }}$ with the beam quality correction applied were obtained for the same reference dosimeters regardless the phantom medium, i.e., $\mathrm{CC} 01$ (6 MeV) and $\mathrm{CC} 13$ $(9 \mathrm{MeV})$, respectively. The differences are larger if various media are used for calibration and dose measurements purposes: $-2.4 \%-3.3 \%$ if the calibration and measurement medium is RW3 solid phantom,

$-3.1 \%-3.8 \%$ if the calibration was done in water and the dose is measured in solid RW3 phantom

$-3.1 \%-5.4 \%$ if the calibration was done in RW3 solid phantom and the dose is measured in water medium.
These differences could be decreased about twice when the $6 \mathrm{MeV}$ beam is replaced by a $9 \mathrm{MeV}$ beam for the crosscalibration procedure.

The precision of cross-calibration procedure performed in solid phantom is lower than that carried out in water medium, despite of the use of $c_{\mathrm{pl}}$ and $\mathrm{h}_{\mathrm{pl}}$ rescaling factors recommended by TRS-398 ${ }^{1}$. However, the use of a solid phantom as the medium for cross calibration procedure of plane-parallel ionization chambers is allowed in some dosimeter testing guidelines $^{\mathbf{2 6}}$, and despite its usage is acceptable for beam of $\mathrm{E} \leq$ $10 \mathrm{MeV}$, it is cautiously recommended for the purpose of absolute dosimetry ${ }^{22,24}$. In this case, the exact determination of perturbation factors is crucial, however, such data are limited in the literature to a few most commonly used materials. The values for other material densities have an accuracy limited to about $1.8 \%$ (based on the linear interpolation procedure), which significantly influences the absolute dose measurements uncertainty.

Despite of extensive interest of the issue of cross-calibration procedure and perturbation factors influencing this process in the past twenty years, the literature still suffers the lack of information regarding some dosimeters which are commercially available and are in clinical use. Therefore studies presenting both, the pattern of such procedure according to the wellestablished and widely used dosimetric protocols, and the quantitative analysis of achieved precision should still be of interest for medical physicists, having particular equipment for use in daily clinical practice.

\section{References}

1. Andreo P, Burns DT, Hohlfeld K, Huq MS, Kanai T, Laitano F, Smyth V, Vynckier S. Absorbed Dose Determination in External Beam Radiotherapy: An International Code of Practice for Dosimetry based on Standards of Absorbed Dose to Water. Vienna: International Atomic Energy Agency. (IAEA TRS-398); 2006.

2. Almond PR, Biggs PJ, Coursey BM, Hanson WF, Huq MS, Nath R, Rogers DWO. AAPM's TG-51 protocol for clinical reference dosimetry of high-energy photon and electron beams. Med Phys. 1999;26(9):1847-1870. https://doi.org/10.1118/1.598691

3. Thwaites DI, DuSautoy AR, Jordan T, McEwen MR, Nisbet A, Nahum AE, Pitchford WG. The IPEM code of practice for electron dosimetry for radiotherapy beams of initial energy from 4 to $25 \mathrm{MeV}$ based on an absorbed dose to water calibration. Phys Med Biol. 2003;48(18):2929-70. https://doi.org/10.1088/0031-9155/48/18/301

4. Deutches Institut für Normung, Procedures of dosimetry with probe-type detectors for photon and electron radiation - Part 2: ionization chamber dosimetry of high energy photon and electron radiation; 2019. DIN 6800-2:2019-07

5. Gerbi BJ, Antolak JA, Deibel FC, Followill DS, Herman MG, Higgins PD, Huq MS, Mihailidis DN, Yorke ED. Recommendations for clinical electron beam dosimetry: supplement to the recommendations of task group 25. Med Phys. 2009;36(7):3239-79. https://doi.org/10.1118/1.3125820

6. Muir BR, McEwen MR. Technical note: On the use of cylindrical ionization chambers for electron beam reference dosimetry. Med Phys. 2017;44(12):6641-46. https://doi.org/10.1002/mp.12582

7. Huq MS. Practical implementation of TG-51. 2003; Retrieved from https://www.aapm.org/meetings/02AM/pdf/8315-36141.pdf

8. Bulski W, Ulkowski P, Gwiazdowska B. Analysis of calibration coefficients of plane-parallel Markus type ionization chambers calibrated in Co-60 and electron beams. Pol J Med Phys Eng. 2007;13(3):163-174. https://doi.org/10.2478/v10013-007-0015-4

9. Christ G, Dohm OS, Bruggmoser G, Schuele E. The use of plane-parallel chambers in electron dosimetry without any cross-calibration. Phys Med Biol. 2002;47(9):N121-N126. https://doi.org/10.1088/0031-9155/47/9/402 
10. Wegener S, Sauer OA. The effective point of measurement for depth-dose measurements in small MV photon beams with different detectors. Med Phys. 2019;46(11):5209-5215. https://doi.org/10.1002/mp.13788

11. Looe HK, Harder D, Poppe B. Experimental determination of the effective point of measurement for various detectors used in photon and electron beam dosimetry. Phys Med Biol. 2011;56(14):4267-4290. https://doi.org/10.1088/0031-9155/56/14/005

12. Zink K, Wulff J. Positioning of a plane-parallel ionization chamber in clinical electron beams and the impact on perturbation factors. Phys Med Biol. 2009;54(8):2421-2435. https://doi.org/10.1088/0031-9155/54/8/011

13. Wang LL, Rogers DWO. Study of the effective point of measurement for ion chambers in electron beams by Monte Carlo simulation. Med Phys. 2009;36(6):2034-2042. https://doi.org/10.1118/1.3121490

14. Anusionwu PC, Alpuche Aviles JE, Pistorius S. The use of 0.5rcav as an effective point of measurement for cylindrical chambers may result in a systematic shift of electron percentage depth doses. J Appl Clin Med Phys. 2020;21(1):117-126. https://doi.org/10.1002/acm2.12797

15. Das IJ, McNeeley SW, Cheng ChW. Ionization chamber shift correction and surface dose measurements in electron beams. Phys Med Biol. 1998;43(11):3419-24. https://doi.org/10.1088/0031-9155/43/11/016

16. Andreo P, Almond PR, Mattsson O, Nahum AE, Roos M. The use of plane-parallel ionization chambers in high-energy electron and photon beams. An international code of practice for dosimetry. Vienna: International Atomic Energy Agency. (IAEA TRS-381); 1995.

17. Roos M, Derikum K, Krauss A. Deviation of the effective point of measurement of the Markus chamber from the front surface of its air cavity in electron beams. Vienna: International Atomic Energy Agency. (IAEA-TECDOC-1173); 2000.

18. Almond PR, Attix FH, Humphries LJ, Kubo H, Nath R, Goetsch S, Rogers DWO. The calibration and use of plane-parallel ionization chambers for dosimetry of electron beams: an extension of the 1983 AAPM protocol report of AAPM Radiation therapy Committee Task Group No. 39. Med Phys. 1994;21(8):1251-1260. https://doi.org/10.1118/1.597359

19. McEwen M, DeWerd L, Ibbott G, Followill D, Rogers DWO, Seltzer S, Seuntjens J. Addendum to the AAPM's TG-51 protocol for clinical reference dosimetry of high-energy photon beams. Med Phys. 2014;41(4):041501. https://doi.org/10.1118/1.4866223

20. Muir BR, Rogers DWO. Monte Carlo calculations of electron beam quality conversion factors for several ion chamber types. Med Phys. 2014;41(11):111701. https://doi.org/10.1118/1.4893915

21. IAEA-TECDOC-1455 Implementation of the International Code of Practice on Dosimetry in Radiotherapy (TRS 398): Review of testing results. Vienna: International Atomic Energy Agency; 2005.

22. Absolute dose measurements in external beam radiotherapy. Application of codes of practice based on standards of absorbed dose to water. IBA Dosimetry GmbH, Schwarzenbruck; 2012.Doc-Id: P-Codes of Practice Absolute Dosimetry-510-001 01.

23. Zakaria A, Schuette W, Younan C. Reference Dosimetry according to the New German Protocol DIN 6800-2 and Comparison with IAEA TRS 398 and AAPM TG 51. Biomed Imaging Interv J. 2011;7(2):e15. https://doi.org/10.2349/biij.7.2.e15

24. SSRPM, Reference dosimetry of high-energy therapy electron beams with ionization chambers. Recommendations No. 10, Revision 2019. ISBN 3908125618.

25. Muir BR. A modified formalism for electron beam reference dosimetry to improve the accuracy of linac output calibration. Med Phys. 2020;47(5):2267-2276. https://doi.org/10.1002/mp.14048

26. Morawska-Kaczyńska M. Anality control of plane-parallel ionization chambers. Recommendations of the Second Standard Dosimetry Laboratory for radiotherapy departments in Poland (in Polish). Nowotwory Journal of Oncology. 2000;50(3):294-302. 